\section{MicroRNAs coordinate an alternative splicing network during mouse postnatal heart development}

\author{
Auinash Kalsotra, ${ }^{1}$ Kun Wang, ${ }^{2}$ Pei-Feng Li, ${ }^{2}$ \\ and Thomas A. Cooper ${ }^{1,3,4}$ \\ ${ }^{1}$ Department of Pathology and Immunology, Baylor College \\ of Medicine, Houston, Texas 77030, USA; ${ }^{2}$ Division of \\ Cardiovascular Research, National Key Laboratory of \\ Biomembrane and Membrane Biotechnology, Institute of \\ Zoology, Chinese Academy of Sciences, Beijing 100101, \\ People's Republic of China; ${ }^{3}$ Department of Molecular and \\ Cellular Biology, Baylor College of Medicine, Houston, Texas \\ 77030, USA
}

\begin{abstract}
Alternative splicing transitions have been identified recently as a conserved component of vertebrate heart remodeling during postnatal development. Here we report that the targeted deletion of Dicer, specifically in adult mouse myocardium, reveals the role of microRNAs (miRNAs) in regulating networks of postnatal splicing transitions and in maintaining adult splicing programs. We demonstrate a direct role for $\mathrm{miR}-23 \mathrm{a} / \mathrm{b}$ in the dramatic postnatal down-regulation of CUGBP and ETR-3-like factor (CELF) proteins that regulate nearly half of developmentally regulated splicing transitions in the heart. These findings define a hierarchy in which rapid postnatal upregulation of specific miRNAs controls expression of alternative splicing regulators and the subsequent splicing transitions of their downstream targets.
\end{abstract}

Supplemental material is available at http://www.genesdev.org.

Received December 7, 2009; revised version accepted February 17, 2010.

MicroRNAs (miRNAs) are key components of posttranscriptional gene regulation with diverse roles in tissue development, differentiation, and homeostasis (van Rooij et al. 2007; Zhao et al. 2007; Bartel 2009). Mature miRNAs are formed by two sequential processing reactions: Primary transcripts of miRNA genes are first cleaved into hairpin-containing intermediates (pre-miRNAs) by the Drosha microprocessor complex, and pre-miRNAs are then processed into mature miRNAs by Dicer (Kim 2005). While miRNA-mediated regulation stimulates quantitative changes in protein and mRNA expression (Baek et al. 2008; Selbach et al. 2008), alternative pre-mRNA splicing controls qualitative gene output in the expression of diverse mRNA isoforms (Blencowe 2006). Recent genome-wide studies revealed that $>90 \%$ of human intron-containing

[Keywords: Alternative splicing networks; CELF proteins; heart development; microRNA]

${ }^{4}$ Corresponding author.

E-MAIL tcooper@bcm.edu; FAX (713) 798-5838.

Article published online ahead of print. Article and publication date are online at http://www.genesdev.org/cgi/doi/10.1101/gad.1894310. genes are alternatively spliced, and more than half of alternative splicing events differ between tissues, revealing an extensive level of regulation (Castle et al. 2008; Pan et al. 2008; Wang et al. 2008).

The mammalian heart undergoes a period of dramatic remodeling during the first 3 wk after birth that requires transcriptional and post-transcriptional regulatory programs not yet fully understood (Xu et al. 2005; Olson 2006). We recently identified a conserved set of alternative splicing transitions during mouse heart development, and demonstrated that nearly half are responsive to the CUGBP and ETR-3-like factor (CELF) family of splicing regulators (Kalsotra et al. 2008). Two CELF proteins, CUGBP1 and CUGBP2, are highly expressed in the fetal heart, but are down-regulated $>10$ fold within 3 wk after birth without a change in mRNA levels.

Here we used targeted deletion of the Dicer gene specifically in adult mouse myocardium to test the role of miRNAs in the mechanism of CELF protein downregulation during postnatal development. Both CELF proteins were dramatically up-regulated within $2 \mathrm{~d}$ of Dicer knockout, as was a subset of five other splicing regulators, while spliceosomal components and an additional set of splicing regulators were not affected. Using multiple independent assays, including antagomir delivery to adult mice, we demonstrate a direct role for miR$23 \mathrm{a} / \mathrm{b}$ in the postnatal down-regulation of the two CELF proteins. Using a data set of postnatal splicing transitions for which we identified cognate regulators, we demonstrate splicing networks that are and are not miRNAdependent in both the Dicer knockout and antagomirtreated hearts. The effects of the Dicer knockout are specific, as neither the expression of splicing regulators nor their target splicing events are affected in three different mouse models of cardiomyopathy. Our results define an important hierarchy in which miRNA expression controls expression of alternative splicing regulators during a critical period of heart development.

\section{Results and Discussion}

The two CELF paralogs expressed in the heart, CUGBP1 and CUGBP2, exhibit a respective 10-fold and 18-fold decrease in protein during the first $2 \mathrm{wk}$ after birth without a decrease in mRNA levels, indicative of posttranscriptional regulation (Kalsotra et al. 2008). CUGBP1 is stabilized by phosphorylation, which decreases during postnatal heart development (Supplemental Fig. 1; Kuyumcu-Martinez et al. 2007). In contrast, CUGBP2 exhibits no change in its phosphorylation state during postnatal development, indicating a different mechanism for its down-regulation (Supplemental Fig. 1).

To determine whether miRNAs are required for repression of CUGBP1 and CUGBP2 proteins in adult hearts, we generated tamoxifen (Tam)-inducible, heart-specific Dicer knockout mice using a Cre-loxP approach (Supplemental Fig. 2A). Mice contain a single Dicer gene that is required to generate mature miRNAs (Birchler and Kavi 2008). Inducible ablation of Dicer in adult hearts overcame previously described early developmental defects (Chen et al. 2008) and allowed timed analysis to distinguish primary effects from secondary compensatory changes. PCR analysis on DNA from heart tissue of Dicer 
homozygous floxed (f/f); MerCreMer (MCM) mice showed recombination only upon Tam treatment, indicating minimal recombination in uninduced mice (Supplemental Fig. 2B). Western blot analysis showed a more than threefold decrease in Dicer protein $8 \mathrm{wk}$ following Tam treatment (Fig. 1A). The loss of mature miRNAs and accumulation of unprocessed precursors confirmed the functional loss of Dicer (Fig. 1B). Residual levels of Dicer protein and mature miRNAs (Fig. 1A,B) in recombined heart tissue were most likely from noncardiomyocytes as well as incomplete recombination and/or long-lived miRNAs.

The cardiomyocyte-specific loss of Dicer in adult mice resulted in dilated cardiomyopathy and premature death within $10 \mathrm{wk}$ of Tam treatment (Fig. 1C). Histology and echocardiography studies revealed dilation of the left ventricle, thinning of the left ventricular wall, and reduced fractional shortening accompanied by systolic and diastolic dysfunction in Dicer ${ }^{\mathrm{f} / \mathrm{f}}$; MCM mice treated with Tam (Fig. 1D; Supplemental Fig. 3A-D). We also detected profound myofiber disarray (Fig. 1E), increased heartto-body weight ratios, widespread apoptosis (Supplemental Fig. 3E,F), myocyte hypertrophy, inflammatory cell infiltration, and focal areas of mild interstitial fibrosis (data not shown). Tam-treated Dicer ${ }^{\mathrm{f} / \mathrm{f}}$ mice and MCM controls showed no changes in several parameters examined (Supplemental Fig. 4A,B; data not shown). Similar effects have been described $1 \mathrm{wk}$ after Tam induction /da Costa Martins et al. 2008).

Consistent with a role for miRNA-mediated repression in the adult heart, CUGBP1 and CUGBP2 proteins were strongly up-regulated in heart tissue from Dicer knockout mice 8 wk following Tam treatment compared with uninduced mice (Fig. 1F). To determine whether CELF protein up-regulation was a general response to cardiac injury, we assayed CUGBP1 and CUGBP2 protein levels in three different mouse models of heart disease (Sano et al. 2002; Braz et al. 2004). While each of these models exhibited altered expression of marker genes, CUGBP1 and CUGBP2 levels were unaffected (Supplemental Fig. 4C). Consistent with elevated CELF protein expression in Dicer knockout hearts, previously identified CUGBP1 target exons that switch their splicing pattern during normal postnatal heart development (Kalsotra et al. 2008) reverted to the embryonic pattern, but were unaffected in the other three models of heart disease (Fig. 1G; Supplemental Fig. 4D). Several constitutive and alternative exons that are not regulated during postnatal heart development showed no changes in Dicer knockout hearts (Supplemental Fig. 5). We conclude that CELF protein induction and the subsequent reversion to embryonic splicing patterns is due to the loss of miRNAs rather than a secondary response to cardiac injury.

We found that CUGBP1 and CUGBP2 up-regulation in Dicer knockout hearts was relatively rapid, and was observed within $48 \mathrm{~h}$ after the last dose of Tam injections (Fig. 2A). Real-time RT-PCR results (Fig. 2B) confirmed that CUGBP1 and CUGBP2 mRNA levels in Dicer knockout hearts $48 \mathrm{~h}$ after Tam treatment remain constant despite striking changes in protein expression. Intriguingly, several other splicing regulators were also up-regulated rapidly after Dicer loss, including Fox-1, Fox-2, PTB, Tia1, and hnRNP H (Fig. 2A). Fox-2 showed a dramatic change in the ratio of its protein isoforms in response to the loss of Dicer, suggestive of a change in splicing pattern. In contrast, the expression of MBNL1, ASF/SF2, hnRNP C, and hnRNP L splicing regulators was unaffected (Fig. 2A).

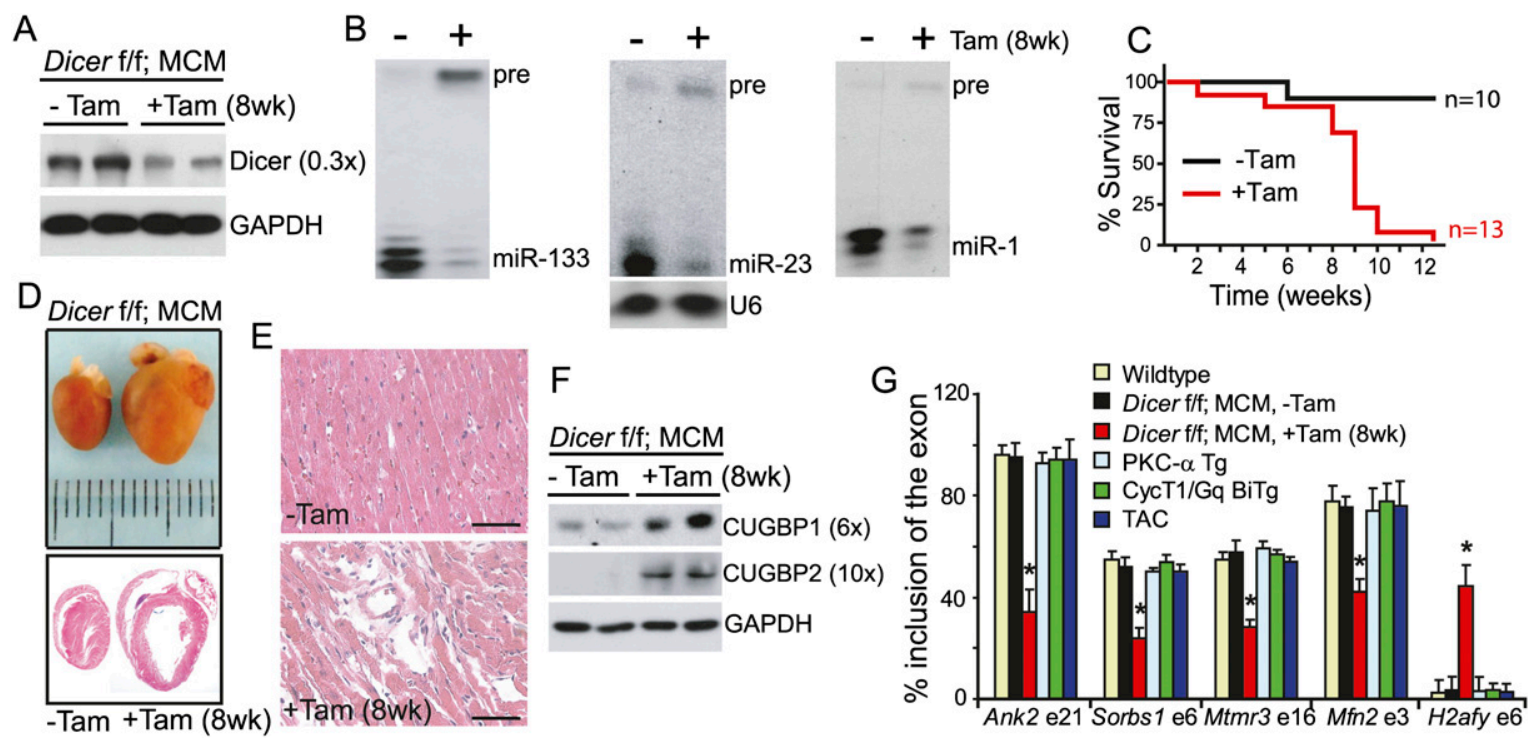

Figure 1. Cardiac-specific deletion of Dicer results in dilated cardiomyopathy and initiates an embryonic splicing program in the adult heart. (A) Western blot showing decrease in Dicer protein in Dicer knockout hearts. Fold decrease in Dicer protein (shown in parentheses) was determined by relative quantification of band intensities, normalized to GAPDH. (B) Northern blots showing depletion of mature miRNAs and accumulation of pre-miRNAs in Dicer knockout hearts. U6 snRNA is the loading control. (C) Kaplan-Meier survival curves for Dicer ${ }^{\mathrm{f} / \mathrm{f}}$; MCM mice with and without Tam treatment. $(D, E)$ Gross morphology and representative images of H\&E staining of transverse sections of hearts from $D_{i c e r}{ }^{f / f}$; MCM mice with and without Tam treatment. $(F)$ Western blots showing increase in steady-state levels of CUGBP1 and CUGBP2 proteins in Dicer knockout hearts. (G) Misregulation of CUGBP1 target exons in Dicer knockout hearts but not in other models of heart disease such as PKC- $\alpha$ transgenic $(\mathrm{Tg})$ mice, CycT1/Gq bitransgenic (BiTg) mice, or wild-type mice after 8 wk of transverse aortic constriction (TAC). Each bar represents mean \pm SD (of four biological replicates) for the percent inclusion of the specified exon. $P<0.05$; asterisk $\left(^{\star}\right)$ indicates significantly different from wild-type mice. 


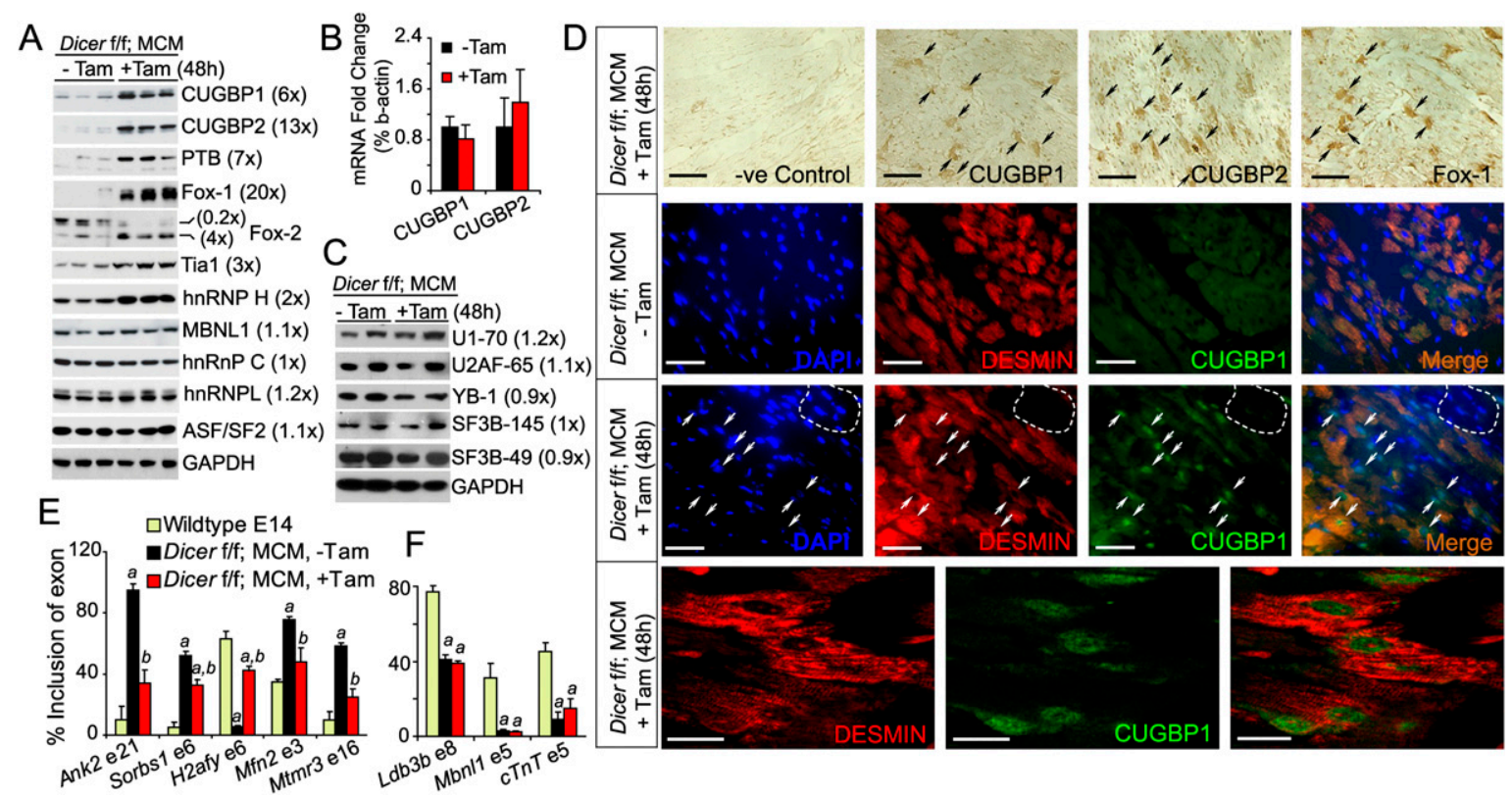

Figure 2. Up-regulation of a subset of splicing regulators in Dicer knockout hearts occurs early and specifically in cardiomyocytes. (A) Steadystate protein levels of alternative splicing regulators. Fold changes are shown in parentheses. $(B)$ CUGBP1 and CUGBP2 mRNA levels, detected by real-time RT-PCR, were unaffected by Dicer knockout. (C) Steady-state protein levels of basal spliceosomal factors. (D) Induced splicing factors in Dicer knockout hearts localize to cardiomyocyte nuclei. The top panel demonstrates immunoreactive nuclei (DAB staining) for CUGBP1, CUGBP2, and Fox-1, as indicated by black arrows. CUGBP1 expression in Dicer knockout hearts was elevated only in the nuclei of cells that costained with desmin (white arrows) and not in the nuclei of other cells (white outline). Cell nuclei were stained with DAPI. All images were taken using the same exposure time. Original magnification: $C$, top three panels, $\times 40 ; C$, bottom panel, $\times 63$. Bar, $50 \mu \mathrm{m}$. Embryonic reversion of splicing for CUGBP1 target exons $(E)$ but not for MBNL1 target exons $(F)$ in adult hearts of Dicer knockouts. $(P<0.05)$. $(a)$ Significantly different from E14; (b) significantly different from Dicer $^{\mathrm{f} / \mathrm{f}} ; \mathrm{MCM}-\mathrm{Tam}$.

In addition, none of the basal spliceosomal components tested showed a change in steady-state protein levels in Dicer knockout hearts (Fig. 2C). Therefore, these results identified a specific set of auxiliary splicing factors that are under regulatory control of miRNAs in the mouse heart.

Inflammatory cells were detected by $48 \mathrm{~h}$ after Dicer loss (data not shown), and we considered the possibility that these infiltrating cells were the source of the increased splicing factors. Immunohistochemistry for CUGBP1, CUGBP2, and Fox-1 in Dicer knockout hearts $48 \mathrm{~h}$ after Tam treatment demonstrated nuclear localization (Fig. 2D, black arrows, top panel). Coimmunofluorescent staining of CUGBP1 with the cardiomyocyte marker desmin confirmed that CUGBP1 protein was elevated only in the nuclei of cardiomyocytes (Fig. 2D, white arrows) but not other cell types (Fig. 2D, white circle, third panel from top). CUGBP1 expression in Dicer ${ }^{\mathrm{f}}$; MCM mice without Tam treatment remained low (Fig. 2D, second panel from top). Thus, we conclude that the rapid up-regulation of specific splicing factors following Dicer gene ablation is specific to cardiomyocytes.

To determine whether early up-regulation of CELF proteins resulted in the embryonic splicing program in adult hearts, we examined splicing of five CUGBP1-regulated exons along with three MBNL1-regulated exons as controls for a splicing regulator not affected by Dicer knockout. As shown in Figure 2, E and F, all eight splicing events underwent robust splicing shifts between embryonic day 14 (E14) and the adult heart. However, only splicing events regulated by CUGBP1 and not those regulated by MBNL1 shifted to the embryonic pattern in Dicer knockout hearts, consistent with the respective changes in protein expres- sion (Fig. 2A,E,F). These results provide strong evidence that, by regulating key splicing factors, miRNAs coordinate developmental transitions in alternative splicing in the postnatal heart.

miRNA-based down-regulation of CELF protein expression during postnatal development could result from either a switch to longer $3^{\prime}$-untranslated regions (UTRs) introducing new miRNA-binding sites (Sandberg et al. 2008; Ji et al. 2009), or up-regulation of specific miRNAs that target CUGBP1 and CUGBP2 mRNAs. Northern blot analysis of CUGBP1 and CUGBP2 mRNAs from E14 and adult mouse heart RNA revealed the absence of large structural changes in these mRNAs during development (Supplemental Fig. 6). Three different algorithms were used to identify candidate miRNAs that potentially regulate CUGBP1 and CUGBP2 expression. A number of miRNAs targeting both CUGBP1 and CUGBP2 were predicted by each program, but only miR-96 and miR-23 were predicted by all three algorithms. Consistent with earlier reports /Chen et al. 2008), we found that miR-96 is not expressed in the heart (Fig. 3B), and therefore focused on miR-23. miR-23 includes miR-23a and miR-23b, which are transcribed from different chromosomes, have identical seed sequences, and differ by only 1 nucleotide on their $3^{\prime}$ ends. There are three conserved miR-23a/b seed matches in the $3^{\prime}$-UTR of CUGBP1 and two in CUGBP2 (Fig. 3A). Strikingly, miR-23a/b expression displays an inverse temporal relationship to CUGBP1 and CUGBP2 protein expression during a narrow period of postnatal heart development. For instance, between postnatal days 6 and 14, miR-23a/b levels increase nearly fourfold, whereas CUGBP1 and CUGBP2 levels decline nearly fivefold and fourfold, respectively (Fig. 3B,C). 


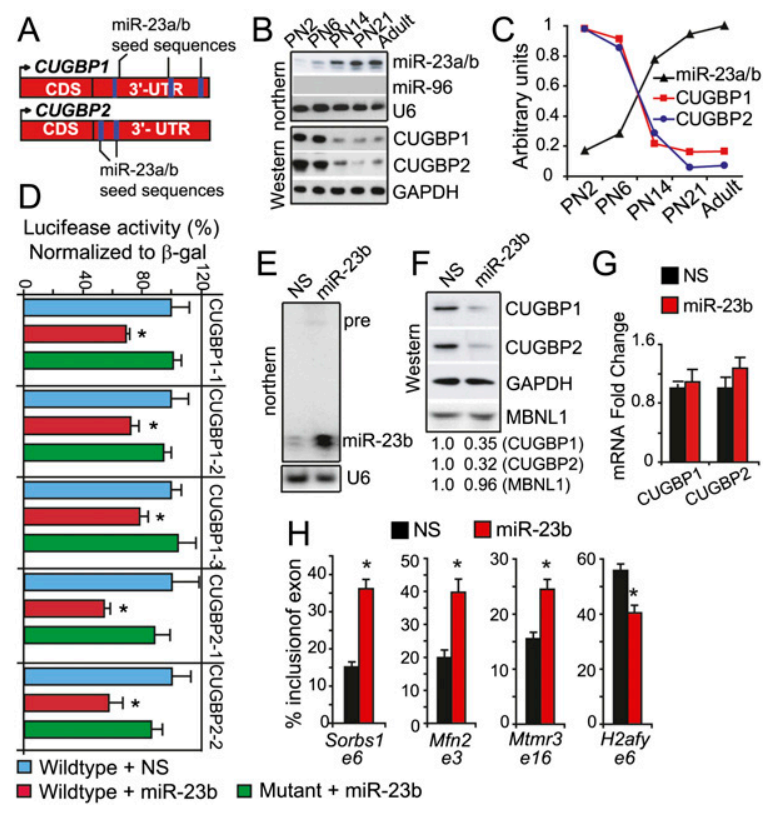

Figure 3. miR-23a/b coordinates the developmental loss of CELF proteins by targeting multiple conserved sites in their $3^{\prime}$-UTRs. (A) Multiple putative miR-23a/b seed elements located in the $3^{\prime}$-UTRs of CUGBP1 and CUGBP2 mRNAs. $(B)$ miR-23a/b exhibits a reciprocal expression pattern to CUGBP1 and CUGBP2 proteins during a narrow time frame of postnatal heart development. $(C)$ Quantitation of steady-state levels of miR-23a/b, CUGBP1, and CUGBP2 proteins. (D) Relative luciferase activity derived from CUGBP1 and CUGBP2 $3^{\prime}$-UTR reporter constructs transfected in HEK293T cells after overexpression of NS or miR-23b. All values are normalized to $\beta$-Gal activity produced from a cotransfected control plasmid. $(E)$ Northern blot analysis demonstrating increased mature miR-23b levels in HL-1 cells infected with miR-23b lentivirus. $(F)$ Significant decrease in CUGBP1 and CUGBP2 proteins in HL-1 cardiomyocytes upon miR$23 \mathrm{~b}$ overexpression. MBNL1 protein levels remain unchanged. Relative quantification of band intensities, normalized to GAPDH levels, are shown below blots. $(G)$ Steady-state mRNA levels of CUGBP1 and CUGBP2 by real-time RT-PCR show no significant change after miR-23b overexpression in HL-1 cells. $(H)$ Splicing of CUGBP1 target exons in HL-1 cells shifts from embryonic to adult patterns upon miR-23b overexpression. $P<0.05$; asterisk $\left({ }^{\star}\right)$ indicates significantly different from NS.

To determine whether CUGBP1 and CUGBP2 transcripts are regulated directly by $\mathrm{miR}-23 \mathrm{a} / \mathrm{b}$, we constructed reporter plasmids in which $3^{\prime}$-UTR segments containing each of the three (CUGBP1) or two (CUGBP2) predicted binding sites were cloned downstream from a luciferase ORF, with or without mutations that would disrupt the predicted miRNA interaction (Supplemental Fig. 7). We overexpressed miR-23b or a nonsilencing control miRNA (NS) in human embryonic kidney (HEK) 293T cells using lentiviral constructs that coexpress TurboRed fluorescent protein. TurboRed fluorescence indicated a high efficiency of infection (Supplemental Fig. 8A) and miR-23b overexpression was confirmed using Northern blots (Supplemental Fig. 8B). miR-23b significantly repressed luciferase activity of each of the five constructs containing wild-type CUGBP1 and CUGBP2, but not the mutant reporter constructs, while cotransfection with NS had no effect (Fig. 3D). These results demonstrate a direct functional interaction between miR-23b and each of these regions within the 3 '-UTR of CUGBP1 and CUGBP2.
To determine whether miR-23b can suppress endogenous CELF protein expression, we overexpressed miR-23b in HL-1 mouse cardiomyocytes (Claycomb et al. 1998). Both CUGBP1 and CUGBP2 are abundantly expressed in these cells, consistent with low expression of endogenous miR-23a/b and expression of the embryonic splicing program for nine out of the 10 developmentally regulated and CUGBP1-responsive splicing events that were screened (data not shown). We overexpressed miR-23b or NS using a lentivirus expression vector (Fig. 3E), and assayed steadystate protein and mRNA levels for CUGBP1 and CUGBP2 by Western blotting and real-time RT-PCR, respectively. CELF protein levels (Fig. 3F), but not mRNA levels (Fig. 3G), were significantly decreased in the presence of miR$23 \mathrm{~b}$ when compared with NS. Consistent with protein down-regulation, CUGBP1 pre-mRNA targets shifted from embryonic to adult splicing patterns in HL-1 cells overexpressing miR-23b (Fig. 3H). Furthermore, miR-23b and miR-23a overexpression in HEK293T cells also resulted in strong down-regulation of CELF protein, demonstrating that $\mathrm{miR}-23$ regulation of CELF proteins is conserved between mice and humans, and suggesting redundancy between miR-23a and miR-23b in regulation of CELF proteins (Supplemental Figs. 8C, 9).

To test whether miR-23a/b are necessary for suppressing CELF protein expression in adult hearts, we infused a miR23a or a negative control (NC) antagomir in wild-type adult mice for systemic delivery to the heart as described previously (Lin et al. 2009). The 25 and $75 \mathrm{mg} / \mathrm{kg}$ doses of miR-23a antagomir produced $50 \%$ and $65 \%$ knockdowns, respectively, of both $\mathrm{miR}-23 \mathrm{a}$ and $\mathrm{miR}-23 \mathrm{~b}$ without causing any overt phenotype (Fig. 4A; data not shown). Administration of the miR-23a antagomir resulted in a significant increase in CUGBP1 and CUGBP2 protein levels in

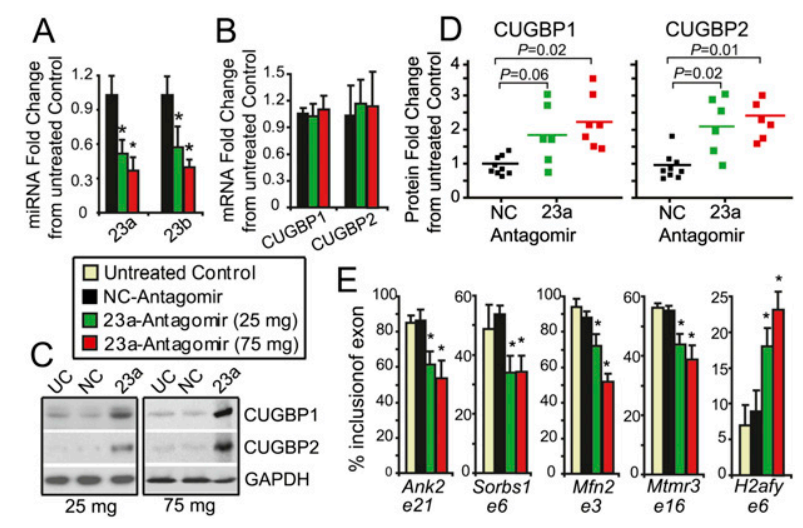

Figure 4. miR-23a/b knockdown in adult mouse hearts reverses CUGBP1 and CUGBP2 protein repression and induces embryonic splicing patterns of CELF targets. $(A)$ Knockdown efficiency of miR$23 \mathrm{a}$ and $\mathrm{miR}-23 \mathrm{~b}$ in adult hearts after miR-23a antagomir treatment ( 25 or $75 \mathrm{mg} / \mathrm{kg}$ per day) was determined by real-time RT-PCR. $(B)$ Steady-state mRNA levels of CUGBP1 and CUGBP2 assayed by real-time RT-PCR show no significant change after miR-23a/b knockdown. (C) Representative Western blots showing CUGBP1 and CUGBP2 up-regulation in adult hearts after miR-23a antagomir treatment. $(D)$ Relative quantitation of CUGBP1 and CUGBP2 levels normalized to GAPDH. (E) Embryonic shift in splicing of CUGBP1 target exons upon miR-23a/b knockdown. Each bar represents mean \pm SD from six wild-type untreated control animals, six wild-type animals treated with NC antagomir, or six wild-type animals treated with miR-23a antagomir. $P<0.05$; asterisk $\left({ }^{\star}\right)$ indicates significantly different from NC anatgomir. 
adult hearts (Fig. 4C,D) without affecting their respective mRNA levels (Fig. 4B). Importantly, the increase in CELF proteins correlated directly with an adult to embryonic shift in the splicing of CUGBP1 pre-mRNA targets (Fig. $4 \mathrm{E})$. In contrast, miR-23a/b knockdown had no effect on the splicing of three MBNL1-regulated exons (Supplemental Fig. 10), demonstrating specificity to CELF pre-mRNA targets. These data indicate that postnatal up-regulation of $\mathrm{miR}-23 \mathrm{a} / \mathrm{b}$ suppresses CUGBP1 and CUGBP2 protein expression in adult hearts via direct binding to their respective $3^{\prime}$-UTRs, resulting in a physiological shift in the alternative splicing of a subset of splicing events.

miRNA-based gene silencing and alternative splicing have emerged as two key post-transcriptional mechanisms that function on a genome-wide scale to regulate tissue development and differentiation (Blencowe 2006; Bartel 2009). The interplay between the miRNA and splicing regulation has been demonstrated in cell culture models in which large-scale changes in splicing occur due to a switch in the expression of splicing regulators $\mathrm{PTB} / \mathrm{nPTB}$ that is controlled by cell- and tissue-specific miRNAs (Boutz et al. 2007; Makeyev et al. 2007). Here we identified a posttranscriptional regulatory network operative during the critical period of postnatal heart development in which coordination of a predominantly conserved set of splicing transitions are driven to the adult pattern, at least in part by miRNA-mediated regulation of splicing factor expression. Most of the auxiliary splicing regulators but none of the basal splicing components examined demonstrated Dicer-dependent regulation, indicating a large potential for linked programs of post-transcriptional regulation. These results also demonstrate that, in addition to modulating splicing transitions during postnatal heart development, miRNAs also maintain appropriate expression of splicing regulators in adult hearts. Disruption of this regulation could cause previously unrecognized mechanisms of disease. For example, aberrant up-regulation of CUGBP1 in adult hearts is associated with myotonic dystrophy in which expression of embryonic splicing patterns in adult tissues lead to specific disease features (Cooper et al. 2009).

\section{Materials and methods}

\section{Animal models}

Mice homozygous for the Dicer ${ }^{\mathrm{f} / \mathrm{f}}$ alleles, were crossed with Tam-inducible MCM transgenic mice that express Cre recombinase protein under the control of the cardiac-specific $\alpha$-myosin heavy-chain (MHC) promoter (Sohal et al. 2001; Yi et al. 2006). Cre-mediated recombination was induced in 2- to 4-mo-old mice by intraperitoneal injection of $20 \mathrm{mg} / \mathrm{kg}$ Tam $/ \mathrm{Tam}$; Sigma-Aldrich) once daily for five consecutive days. Dicer ${ }^{\mathrm{f} / \mathrm{f}} ;$ MCM mice treated with vehicle alone (-Tam) were used as controls. In vivo inhibition of miR-23 was performed by infusing a miR-23a antagomir $(25$ or $75 \mathrm{mg} / \mathrm{kg}$ per day) for 1 wk using Alzet osmotic pumps as described previously (Lin et al. 2009). NC antagomir (5'-AGUACUUUUGUGUAGUACAA-3') served as a NC. All experiments were conducted in accordance with the NIH Guide for the Use and Care of Laboratory Animals and were approved by the Institutional Animal Care and Use Committee of Baylor College of Medicine.

\section{Cell culture and transfections}

HL-1 cells were cultured on gelatin $(0.02 \%, \mathrm{w} / \mathrm{v}) /$ fibronectin $(10 \mu \mathrm{g} / \mathrm{mL})$ coated plates and were maintained in Claycomb medium (JRH Biosciences) supplemented with $10 \%$ fetal bovine serum (JRH Biosciences), $2 \mathrm{mM}$ L-glutamine, $0.1 \mathrm{mM}$ norepinephrine, $100 \mathrm{U} / \mathrm{mL}$ penicillin, and $100 \mu \mathrm{g} / \mathrm{mL}$ streptomycin (Life Technologies) as described previously (Claycomb et al. 1998). HEK293T cells were cultured in high-glucose (4.5 g/L) DMEM supplemented with $10 \%$ fetal bovine serum, $2 \mathrm{mM} \mathrm{L-glutamine,} 1 \times$ sodium pyruvate, $100 \mathrm{U} / \mathrm{mL}$ penicillin, and $100 \mu \mathrm{g} / \mathrm{mL}$ streptomycin. Viral stocks for miR-23b and a NS (contains a random nontargeting sequence; Open Biosystems) were produced using the lentiviral packaging plasmids psPAX2 and PMD2.G (Addgene, Inc.), concentrated and titered as per the manufacturer's instructions. The HL-1 cells were infected in T25 flasks with miR$23 \mathrm{~b}$ or NS viral stocks in the presence of $8 \mu \mathrm{g} / \mathrm{mL}$ polybrene. After $48 \mathrm{~h}$ of selection in $2 \mu \mathrm{g} / \mathrm{mL}$ puromycin selection media, cells were harvested to isolate total RNA or proteins. For transient transfections, miRNA mimics for NS, miR-22, or miR-23a were purchased from Dharmacon and were transfected at $100 \mathrm{nM}$ final concentration using the manufacturer's instructions.

\section{Protein and mRNA expression analysis}

Protein expression by Western blot analysis and RNA expression by semiquantitative and real-time RT-PCR was performed by using standard procedures. Antibody and primer information is provided in the Supplemental Material.

\section{RT-PCR splicing analysis}

Splicing analysis by RT-PCR was performed using unique primers around potential splicing regions as described previously (Kalsotra et al. 2008).

Histology, immunohistochemistry, Northern blotting, luciferase assay, and cardiac function analysis

Details of histology, immunohistochemistry, Northern blotting, luciferase assay, and cardiac function analysis are provided in the Supplemental Material.

\section{Statistics}

Values are presented as mean \pm SD. Statistical significance was determined using two-tailed Student's $t$-test or one-way ANOVA followed by post hoc Tukey's multiple range test $(P<0.05)$.

\section{Acknowledgments}

We thank Dr. A. Tarakhovsky for providing Dicer $\mathrm{f} / \mathrm{f}$ mice, Dr. J. Molkentin for providing MCM mice and heart tissues from PKC $\alpha$-Tg mice, and Dr. M. Schneider and Dr. X. Wehrens for providing heart tissues from CycT1/Gq $\alpha$ BiTg mice and TAC operated mice, respectively. We are also grateful to Dr. W. Claycomb for providing HL-1 cells, and to Dr. S. Anakk for help with luciferase assays. This project was funded by the NIH grant R01GM076493 to T.A.C., Myotonic Dystrophy Foundation Postdoctoral award to A.K., and NNSFC grant G30730045 to P.F.L.

\section{References}

Baek D, Villen J, Shin C, Camargo FD, Gygi SP, Bartel DP. 2008. The impact of microRNAs on protein output. Nature 455: 64-71.

Bartel DP. 2009. MicroRNAs: Target recognition and regulatory functions. Cell 136: 215-233.

Birchler JA, Kavi HH. 2008. Molecular biology. Slicing and dicing for small RNAs. Science 320: 1023-1024.

Blencowe BJ. 2006. Alternative splicing: New insights from global analyses. Cell 126: 37-47.

Boutz PL, Chawla G, Stoilov P, Black DL. 2007. MicroRNAs regulate the expression of the alternative splicing factor $\mathrm{nPTB}$ during muscle development. Genes \& Dev 21: 71-84.

Braz JC, Gregory K, Pathak A, Zhao W, Sahin B, Klevitsky R, Kimball TF, Lorenz JN, Nairn AC, Liggett SB, et al. 2004. PKC- $\alpha$ regulates cardiac contractility and propensity toward heart failure. Nat Med 10: 248-254.

Castle JC, Zhang C, Shah JK, Kulkarni AV, Kalsotra A, Cooper TA, Johnson JM. 2008. Expression of 24,426 human alternative splicing events and predicted cis regulation in 48 tissues and cell lines. Nat Genet 40: 1416-1425.

Chen JF, Murchison EP, Tang R, Callis TE, Tatsuguchi M, Deng Z, Rojas M, Hammond SM, Schneider MD, Selzman CH, et al. 2008. Targeted 


\section{Kalsotra et al.}

deletion of Dicer in the heart leads to dilated cardiomyopathy and heart failure. Proc Natl Acad Sci 105: 2111-2116.

Claycomb WC, Lanson NA Jr, Stallworth BS, Egeland DB, Delcarpio JB, Bahinski A, Izzo NJ Jr. 1998. HL-1 cells: A cardiac muscle cell line that contracts and retains phenotypic characteristics of the adult cardiomyocyte. Proc Natl Acad Sci 95: 2979-2984.

Cooper TA, Wan L, Dreyfuss G. 2009. RNA and disease. Cell 136: 777793.

da Costa Martins PA, Bourajiaj M, Gladka M, Kortland M, van Oort RJ, Pinto YM, Molkentin JD, De Windt LJ. 2008. Conditional dicer gene deletion in the postnatal myocardium provokes spontaneous cardiac remodeling. Circulation 118: 1567-1576.

Ji Z, Lee JY, Pan Z, Jiang B, Tian B. 2009. Progressive lengthening of 3' untranslated regions of mRNAs by alternative polyadenylation during mouse embryonic development. Proc Natl Acad Sci 106: 70287033.

Kalsotra A, Xiao X, Ward AJ, Castle JC, Johnson JM, Burge CB, Cooper TA. 2008. A postnatal switch of CELF and MBNL proteins reprograms alternative splicing in the developing heart. Proc Natl Acad Sci 105: 20333-20338.

Kim VN. 2005. MicroRNA biogenesis: Coordinated cropping and dicing. Nat Rev Mol Cell Biol 6: 376-385.

Kuyumcu-Martinez NM, Wang GS, Cooper TA. 2007. Increased steadystate levels of CUGBP1 in myotonic dystrophy 1 are due to PKCmediated hyperphosphorylation. Mol Cell 28: 68-78.

Lin Z, Murtaza I, Wang K, Jiao J, Gao J, Li PF. 2009. miR-23a functions downstream of NFATc3 to regulate cardiac hypertrophy. Proc Nat1 Acad Sci 106: 12103-12108.

Makeyev EV, Zhang J, Carrasco MA, Maniatis T. 2007. The microRNA miR-124 promotes neuronal differentiation by triggering brain-specific alternative pre-mRNA splicing. Mol Cell 27: 435-448.

Olson EN. 2006. Gene regulatory networks in the evolution and development of the heart. Science 313: 1922-1927.

Pan Q, Shai O, Lee LJ, Frey BJ, Blencowe BJ. 2008. Deep surveying of alternative splicing complexity in the human transcriptome by highthroughput sequencing. Nat Genet 40: 1413-1415.

Sandberg R, Neilson JR, Sarma A, Sharp PA, Burge CB. 2008. Proliferating cells express mRNAs with shortened $3^{\prime}$ untranslated regions and fewer microRNA target sites. Science 320: 1643-1647.

Sano M, Abdellatif M, Oh H, Xie M, Bagella L, Giordano A, Michael LH, DeMayo FJ, Schneider MD. 2002. Activation and function of cyclin $\mathrm{T}-\mathrm{Cdk} 9$ (positive transcription elongation factor-b) in cardiac muscle-cell hypertrophy. Nat Med 8: 1310-1317.

Selbach M, Schwanhausser B, Thierfelder N, Fang Z, Khanin R, Rajewsky N. 2008. Widespread changes in protein synthesis induced by microRNAs. Nature 455: 58-63.

Sohal DS, Nghiem M, Crackower MA, Witt SA, Kimball TR, Tymitz KM, Penninger JM, Molkentin JD. 2001. Temporally regulated and tissuespecific gene manipulations in the adult and embryonic heart using a tamoxifen-inducible Cre protein. Circ Res 89: 20-25.

van Rooij E, Sutherland LB, Qi X, Richardson JA, Hill J, Olson EN. 2007. Control of stress-dependent cardiac growth and gene expression by a microRNA. Science 316: 575-579.

Wang ET, Sandberg R, Luo S, Khrebtukova I, Zhang L, Mayr C, Kingsmore SF, Schroth GP, Burge CB. 2008. Alternative isoform regulation in human tissue transcriptomes. Nature 456: 470-476.

$\mathrm{Xu}$ X, Yang D, Ding JH, Wang W, Chu PH, Dalton ND, Wang HY, Bermingham JR Jr, Ye Z, Liu F, et al. 2005. ASF/SF2-regulated CaMKII alternative splicing temporally reprograms excitation-contraction coupling in cardiac muscle. Cell 120: 59-72.

Yi R, O'Carroll D, Pasolli HA, Zhang Z, Dietrich FS, Tarakhovsky A, Fuchs E. 2006. Morphogenesis in skin is governed by discrete sets of differentially expressed microRNAs. Nat Genet 38: 356-362.

Zhao Y, Ransom JF, Li A, Vedantham V, von Drehle M, Muth AN, Tsuchihashi T, McManus MT, Schwartz RJ, Srivastava D. 2007. Dysregulation of cardiogenesis, cardiac conduction, and cell cycle in mice lacking miRNA-1-2. Cell 129: 303-317. 


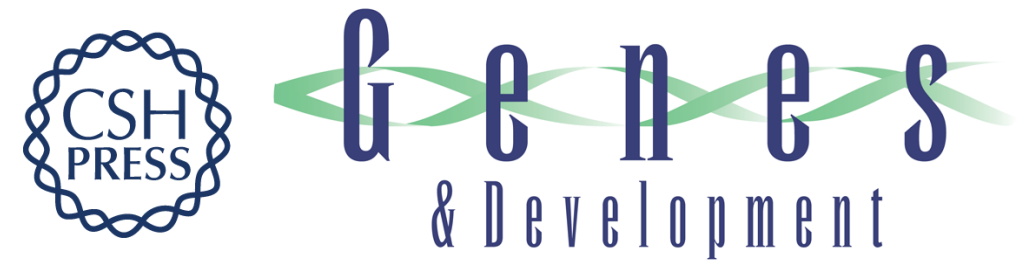

\section{MicroRNAs coordinate an alternative splicing network during mouse postnatal heart development}

Auinash Kalsotra, Kun Wang, Pei-Feng Li, et al.

Genes Dev. 2010, 24: originally published online March 18, 2010

Access the most recent version at doi:10.1101/gad.1894310

\section{Supplemental http://genesdev.cshlp.org/content/suppl/2010/03/10/gad.1894310.DC1 Material}

References This article cites 28 articles, 12 of which can be accessed free at: http://genesdev.cshlp.org/content/24/7/653.full.html\#ref-list-1

\section{License}

Email Alerting

Receive free email alerts when new articles cite this article - sign up in the box at the top Service 